\title{
Game Theoretic Considerations for the Gaussian Multiple Access Channel
}

\author{
Vojislav Gajic and Bixio Rimoldi \\ Mobile Communications Laboratory \\ EPFL \\ Lausanne, Switzerland \\ \{vojislav.gajic, bixio.rimoldi\}@epfl.ch
}

\begin{abstract}
We study the behavior of users in a classical Additive White Gaussian Noise Multiple Access Channel. We model users as rational entities whose only interest is to maximize their own communication rate, and we model their interaction as a noncooperative one-shot game. The Nash equilibria of the two-user game are found, and the relation between the purestrategy and mixed-strategy Nash equilibria is discussed. As in most games, the absence of cooperation and coordination leads to inefficiencies. We then extend our setting using evolutionary game theory, which we use to model a large population of users playing the MAC game over time. A unique evolutionary stable strategy is found for this case, corresponding to the strategy achieving the Nash equilibrium in a simplified one-shot game. Finally, we investigate what happens to the distribution of strategies in a population when we assume that the number of offsprings of a user is equal to the payoff of this user in a one-shot game. We find that the system converges to a state in which the average strategy of the population is the evolutionary stable strategy.
\end{abstract}

\section{INTRODUCTION}

In this paper we focus on the Additive White Gaussian Noise (AWGN) Multiple Access Channel (MAC). We are interested in describing the behavior of users in a situation where users make independent decisions about their coding rate, assuming that prior offline agreements are not possible and any central authority is absent. This is in contrast to the standard setup that implicitly assumes some sort of coordination in choosing the individual rates.

We adopt a game-theoretic approach [1], assuming rational users whose only interest is to maximize their own communication rate. We propose and analyze a one-shot noncooperative game. For this game, all of the points on the dominant face of the capacity region are Nash equilibria. These equilibria are Pareto optimal (there exist no component-wise better outcome), but it is not clear which one of them would be played. Alternatively, we study randomized strategies, and find the probability distributions that constitute the mixed strategy Nash equilibria. Next, we model the situation where a large number of users interacts through the one-shot AWGN MAC game. The game is played repeatedly by users randomly chosen from a large population, and over a long period of time. We model this situation using evolutionary game theory [2]. We show that the strategy achieving the mixed strategy Nash equilibrium in a simplified one shot game is the unique evolutionary stable strategy when users are playing this game. This means that a population of users playing this strategy would be getting better rates than a mutant population playing any different strategy. Finally, we investigate what happens to the distribution of strategies in a population when we assume that the number of offsprings of a user is equal to the payoff of this user in a one-shot game. We find that the system converges to a state in which the average strategy of the population is the evolutionary stable strategy.

In our model, users are operating under a power constraint over a scalar Gaussian channel. This is in contrast to previous work on a fading channel model, where users can exploit different fading states to reach a sum-rate optimal operating point [3]. In our work, the optimal power allocation of a user is to transmit at maximum power, so power control is not an issue. The strategies of the users then consist in choosing their communication rates, and the receiver's only role is to decode, if possible. Previous work investigated the behavior of users in CDMA ([4], [5], [6] and [7] just to name a few). In this case the rate is calculated directly from the SINR, and the strategy of a user consists in choosing the transmit power. In a similar setting [8], the authors describe how users reach the optimal sum-rate power allocation for the limiting case where the number of users goes to infinity and the choice of the decoding order is up to an arbitrator. In this case the users' choice is again limited to a power allocation. Similarly, in [3], the receiver imposes a specific decoding order, which leads to users adopting optimal power allocations on different degrees of freedom. Once more the users' rates are uniquely determined from the power allocations. Evolutionary game theory has already been applied in a communication setting in [9] and [10]. Finally, behavior of users in the AWGN MAC was investigated in the cooperative game theoretic setting in [11].

\section{Communication Model}

We consider a Gaussian MAC with two senders and one receiver. The signal at the receiver is

$$
Y=X_{1}+X_{2}+Z
$$

where $X_{i} \in \mathbb{R}$ is the transmitted signal of user $i$ and $Z \in \mathbb{R}$ is zero mean Gaussian noise of unit variance. Each user has an individual average input power constraint $\mathbb{E}\left[\left|X_{i}\right|^{2}\right] \leq P_{i}$. The capacity region $\mathcal{C}$ for this channel [12] is the set of all 
rate pairs $\left(R_{1}, R_{2}\right)$ such that

$$
\begin{aligned}
R_{i} & \leq \frac{1}{2} \log \left(1+P_{i}\right), i \in\{1,2\} \\
R_{1}+R_{2} & \leq \frac{1}{2} \log \left(1+P_{1}+P_{2}\right) .
\end{aligned}
$$

We denote by $C_{i}=0.5 \log \left(1+P_{i}\right)$ the capacity of user $i$ and by $r_{i}^{0}=0.5 \log \left(1+\frac{P_{i}}{1+P_{3-i}}\right), i \in\{1,2\}$, the rate of user $i$ when the signal of the other user is treated as noise. Notice that the message of user $i$ is decodable when $r_{i} \leq r_{i}^{0}$ even if the rate pair $\left(r_{1}, r_{2}\right)$ lies outside the capacity region $\mathcal{C}$. This is illustrated by the shaded regions in Figure 1. For obvious reasons we will refer to $r_{i}^{0}$ as the "safe rate".

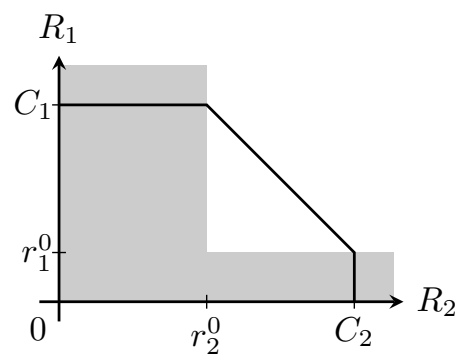

Fig. 1. AWGN MAC Capacity Region

\section{GAme Theoretic Models And Notation}

We assume that users are only interested in maximizing their own communication rate, and that they are aware that the other user has the same goal. The power constraints are also common knowledge. Users have to choose independently their own coding rate at the beginning of the communication, where the rate selected by a user may be either deterministic, or chosen from some distribution. If the chosen rate tuple lies in the capacity region, users will communicate at that operating point. Otherwise, either the receiver is unable to decode any signal and the observed rates are both zero, or only one of the signals can be decoded (if one of the users is transmitting at or below the safe rate). With these assumptions we can define a non-cooperative one-shot game, which we call the Gaussian MAC game. The set of allowed strategies for user $i$ is the set of all probability distributions over $\left[0, C_{i}\right]$, and the payoff is the obtained rate.

We will denote the (random) attempted rate of user $i$ as $R_{i}$, and $r_{i}$ as its sample value. Notice that $r_{i}$ and $R_{i}$ are not the actual rates. If the attempted rates are $r_{1}$ and $r_{2}$, the utility (i.e. actual rate) of user $i$, denoted $u_{i}\left(r_{1}, r_{2}\right)$, is:

$$
u_{i}\left(r_{1}, r_{2}\right)= \begin{cases}r_{i}, & \text { if }\left(r_{1}, r_{2}\right) \in \mathcal{C} \text { or } r_{i} \leq r_{i}^{0} \\ 0, & \text { else. }\end{cases}
$$

We denote the probability density function of user $i$ as $f_{R_{i}}\left(r_{i}\right)=f_{i}\left(r_{i}\right)$. We call $f_{i}(\cdot)$ the mixed strategy of user $i$, and the tuple $\left(f_{1}\left(r_{1}\right), f_{2}\left(r_{2}\right)\right)$ a strategy profile. A mixed strategy that chooses some coding rate $r_{i}$ with probability 1 is called a pure strategy, denoted $\delta_{r_{i}}$. The quantity of interest for the users is their expected utility $\mathcal{U}_{i}\left(f_{1}, f_{2}\right)$, or simply $\mathcal{U}_{i}$, where $\mathcal{U}_{i}\left(f_{1}, f_{2}\right)=\mathbb{E}\left[u_{i}\left(R_{1}, R_{2}\right)\right]$. Since the users are making independent decisions, the joint density of $\left(R_{1}, R_{2}\right)$ is simply the product of the individual densities.

\section{NASH EQUILIBRIA}

We are interested in finding strategy profiles that users participating in this game might adopt. We focus on the concept of Nash equilibrium. Nash equilibrium is a strategy profile for which it is not beneficial for any user to play a different strategy. In general Nash equilibria are desirable operating points since users have no incentive to deviate from them unilaterally. Problems may arise when there is more than one Nash equilibrium in a given game, in which case some other method needs to be used to determine which of these equilibria will be played.

Any pure strategy profile $\left(\delta_{r_{1}}, \delta_{C_{1,2}-r_{1}}\right)$, where $r_{1} \in$ $\left[r_{1}^{0}, C_{1}\right]$ and $C_{1,2}=0.5 \log \left(1+P_{1}+P_{2}\right)$, is a pure-strategy Nash equilibrium of the Gaussian MAC game. These strategy profiles are exactly those for which $\left(r_{1}, r_{2}\right)$ is a point on the dominant face of the capacity region. Therefore the Gaussian MAC game has an infinite number of (pure strategy) Nash equilibria. As discussed in the previous paragraph, it is not clear which of these equilibria would be played. In the remainder of this section we study mixed strategies. A mixed strategy Nash equilibrium is a tuple $\left(f_{1}^{*}, f_{2}^{*}\right)$ of probability density functions such that $\mathcal{U}_{1}\left(f_{1}^{*}, f_{2}^{*}\right) \geq \mathcal{U}_{1}\left(f_{1}, f_{2}^{*}\right)$ and $\mathcal{U}_{2}\left(f_{1}^{*}, f_{2}^{*}\right) \geq \mathcal{U}_{2}\left(f_{1}^{*}, f_{2}\right)$ for all $f_{1}$ and $f_{2}$.

To find mixed strategy Nash equilibria of the Gaussian MAC game, we start with the following observation: a rational user will never transmit at a rate below $r_{i}^{0}$, since that rate can always be improved upon. Therefore, in the mixed strategy equilibrium, user $i$ will have a probability density function of support in $\left[r_{i}^{0}, C_{i}\right]$. We call this interval the set of rational strategies. In Theorem 1 we show that there is a unique Nash equilibrium with the entire set of rational strategies of that user as support set.

The following Lemma reveals the crucial property of a mixed strategy Nash equilibrium.

Lemma 1: Let $g_{1}:\left[r_{1}^{0}, C_{1}\right] \rightarrow \mathbb{R}^{+}$be defined as

$$
g_{1}(r)=\mathbb{E}\left[u_{1}\left(r, R_{2}\right)\right]=\int_{r_{2}^{0}}^{C_{2}} f_{2}(\xi) u_{1}(r, \xi) d \xi,
$$

and, similarly, let $g_{2}(r)=\mathbb{E}\left[u_{2}\left(R_{1}, r\right)\right]$.

For $i \in\{1,2\}$, let $\bar{\lambda}_{i}=\max _{r \in\left[r_{i}^{0}, C_{i}\right]} g_{i}(r)$ and let $\overline{\mathcal{J}}_{i}=g_{i}^{-1}\left(\bar{\lambda}_{i}\right)$. The mixed strategy profile $\left(f_{1}, f_{2}\right)$ is a Nash equilibrium iff

$$
\int_{\overline{\mathcal{J}}_{i}} f_{i}(\xi) d \xi=1, \quad i=1,2
$$

Proof: By definition, the mixed strategy profile $\left(f_{1}, f_{2}\right)$ is a Nash equilibrium iff, for $i=1,2$, the expected utility of user $i$ cannot be increased by unilaterally changing $f_{i}$. Concerning user 1 , observe that

$$
\mathbb{E}\left[u_{1}\left(R_{1}, R_{2}\right)\right]=\int_{r_{1}^{0}}^{C_{1}} g_{1}(r) f_{1}(r) d r \leq \bar{\lambda}_{1}
$$


and that the inequality holds with equality iff (2) is true for $i=1$. Similarly, $\mathbb{E}\left[u_{2}\left(R_{1}, R_{2}\right)\right] \leq \bar{\lambda}_{2}$ with equality iff (2) is true for $i=2$.

We begin by finding the unique mixed strategy Nash equilibrium in which users have positive probability density almost everywhere on the set of rational strategies.

Theorem 1: The following probability distributions constitute the unique Nash equilibrium for which the density of $R_{i}$, for $i \in\{1,2\}$, is strictly positive almost everywhere on the interval $\left[r_{i}^{0}, C_{i}\right]$ :

$$
\begin{aligned}
& \operatorname{Pr}\left\{R_{1} \leq r\right\}= \begin{cases}\frac{r_{2}^{0}}{C_{1,2}-r}, & r \in\left[r_{1}^{0}, C_{1}\right] \\
0, & \text { else }\end{cases} \\
& \operatorname{Pr}\left\{R_{2} \leq r\right\}= \begin{cases}\frac{r_{1}^{0}}{C_{1,2}-r}, & r \in\left[r_{2}^{0}, C_{2}\right] \\
0, & \text { else }\end{cases}
\end{aligned}
$$

Proof: Suppose that there exist $f_{1}$ and $f_{2}$ such that $g_{i}\left(r_{i}\right)=\lambda_{i}$, for all $r_{i} \in\left[r_{i}^{0}, c_{i}\right], i=1,2$, where $\lambda_{i}$ 's are some constants. Then, as an immediate corollary to Lemma 1 , the mixed strategy profile $\left(f_{1}, f_{2}\right)$ is a Nash equilibrium. To find such probability distributions, we begin by noting that $g_{1}\left(r_{1}^{0}\right)=r_{1}^{0}$, regardless of $f_{2}$. Hence, $\lambda_{1}=r_{1}^{0}$. Then, $f_{2}$ needs to be such that $g_{1}\left(r_{1}\right)=r_{1}^{0}$, for all $r_{1} \in\left[r_{1}^{0}, C_{1}\right]$ :

$$
r_{1}^{0}=\lambda_{1}=\mathbb{E}\left[u_{1}\left(r_{1}, R_{2}\right)\right]=r_{1} \operatorname{Pr}\left\{R_{2} \leq C_{1,2}-r_{1}\right\} .
$$

By substituting $C_{1,2}-r_{1}=r, r \in\left[r_{2}^{0}, C_{2}\right]$, we can find the probability distribution of user 2 in the mixed strategy Nash equilibrium:

$$
\operatorname{Pr}\left\{R_{2} \leq r\right\}=\frac{r_{1}^{0}}{C_{1,2}-r}, r \in\left[r_{2}^{0}, C_{2}\right] .
$$

Similarly, we find the probability distribution of user 1 . These probability distribution are unique by construction, hence so is this mixed strategy Nash equilibrium.

Recall that, due to rationality, user $i$ will not choose any rate below $r_{i}^{0}$. On the other hand, (3) evaluated at $r_{1}^{0}$ implies that $\operatorname{Pr}\left\{R_{1} \leq r_{1}^{0}\right\}=\frac{r_{2}^{0}}{C_{2}}$. Therefore,

$$
\operatorname{Pr}\left\{R_{1}=r_{1}^{0}\right\}=\frac{r_{2}^{0}}{C_{2}} .
$$

So, in this mixed strategy Nash equilibrium, a user chooses the safe rate with a positive probability, and the remaining rates in $\left(r_{i}^{0}, C_{i}\right]$ by sampling from a continuous probability distribution.

The following result generalizes Theorem 1.

Theorem 2: Let $f_{i}\left(r_{i}\right)>0$ almost everywhere on $\mathcal{I}^{i} \subset$ $\left[r_{i}^{0}, C_{i}\right], i=1,2$, where $\mathcal{I}^{i}$ is the union of finitely many closed disjoint intervals. Let $a=\min \left\{r_{1} \mid r_{1} \in \mathcal{I}^{1}\right\}, b=$ $\max \left\{r_{1} \mid r_{1} \in \mathcal{I}^{1}\right\}$ and $\mathcal{I}^{2}=\left\{r_{2}\right.$ s.t. $\left.r_{1}+r_{2}=C_{1,2}, r_{1} \in \mathcal{I}^{1}\right\}$. Then, the following probability distributions constitute a Nash equilibrium

$$
\operatorname{Pr}\left\{R_{1} \leq r\right\}= \begin{cases}0, & r<a \\ \frac{C_{1,2}-b}{C_{1,2}-\hat{r}_{1}}, & \text { else }\end{cases}
$$

$$
\operatorname{Pr}\left\{R_{2} \leq r\right\}= \begin{cases}0, & r<C_{1,2}-b \\ \frac{a}{C_{1,2}-\hat{r}_{2}}, & \text { else }\end{cases}
$$

where $\hat{r}_{i}=\max \left\{\tilde{r} \mid \tilde{r} \in \mathcal{I}^{i}\right.$ and $\left.\tilde{r} \leq r\right\}, i=1,2$. An example of such a distribution for one of the users can be seen in Figure 2 , for the case when $\mathcal{I}=\left[r^{0}, r^{1}\right] \cup\left[r^{2}, C\right]$. The proof of this Theorem (omitted for brevity) also relies on Lemma 1.

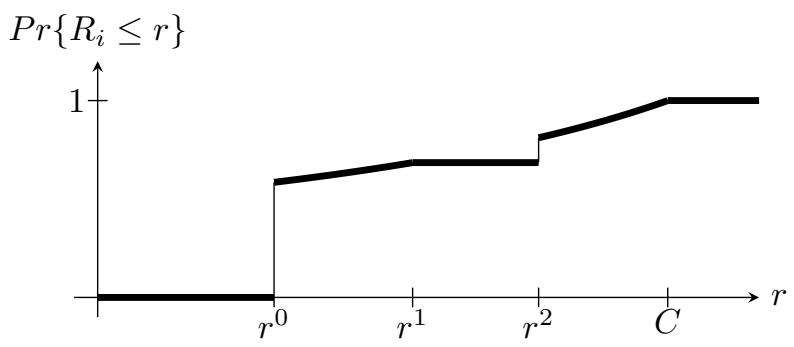

Fig. 2. An example of a probability distribution achieving Nash equilibrium

The result of Theorem 2 seems to be more relevant if the sets $\mathcal{I}^{1}$ and $\mathcal{I}^{2}$ are fixed ahead of time, which is prevented by the no coordination assumption. One exception (when this assumption is automatically true) is when $\mathcal{I}^{1}$ and $\mathcal{I}^{2}$ are $\left[r_{1}^{0}, C_{1}\right]$ and $\left[r_{2}^{0}, C_{2}\right]$, respectively, in which case Theorem 2 reduces to Theorem 1. For this reason we consider the mixed strategy Nash equilibrium of Theorem 1 to be the most interesting one.

The expected payoff of a user in this Nash equilibrium is $\mathcal{U}_{i}=r_{i}^{0}$. One can then wonder why users would not simply transmit at the safe rate with probability 1 , resulting in the outcome $\left(r_{1}^{0}, r_{2}^{0}\right)$. But if this were the case, a user would be tempted to transmit at capacity. Therefore, each user has some incentive to code at the safe rate, and some incentive to try out higher rates with positive probability. This is exactly what is predicted by the mixed strategy Nash equilibrium found in Theorem 1. The price for the lack of cooperation and coordination is that the mixed strategy Nash equilibrium is not Pareto optimal since any point on the dominant face is component-wise better.

\section{Evolutionary Game Theory}

One of the principal assumptions of non-cooperative game theory, considered in the previous section, is user rationality. This implies not only that users are interested in maximizing their payoff, but also that they are able to calculate a precise probability in order to go about their daily communication. In general we would rather expect to observe learning by trial-and-error through many interactions with many different individuals. To study this setting, we introduce a model from evolutionary game theory. Evolutionary game theory assumes that the one-shot noncooperative game is played repeatedly by players that are randomly chosen from a large population of users. Users are considered non-rational in the sense that they are playing pre-programmed strategies that are not necessarily optimal. However, the payoff of a user may change either due 
to a change in strategy of a subpopulation (mutation) or due to the fact that a fraction of a population playing a certain strategy can change (evolution).

In this section we consider both scenarios. We begin by asking if there is a strategy that, if adopted by the entire population, will have a greater payoff than a subpopulation of mutants playing some different strategy. In the second part of the section we study how the distribution of strategies in a population evolves. We pose these questions for the population of users who are all playing a simplified symmetric Gaussian MAC game, where symmetric refers to $P_{1}=P_{2}$.

Formally, we consider a large population of communication users whose power is limited by $P$. Furthermore, let $r^{0}=$ $\log \left(1+\frac{P}{1+P}\right)$ and $C=\log (1+P)$ be the only two allowed coding rates for any user drawn to play the game. Then, the set of allowed distributions is $\Sigma=\left\{f(\cdot): f(r)=\sigma \delta_{r^{0}}+\right.$ $\left.(1-\sigma) \delta_{C}, \sigma \in[0,1]\right\}$. If a fraction $\bar{\epsilon}=1-\epsilon$ of the population is programmed to play strategy $f_{I}=\sigma_{I} \delta_{r^{0}}+\left(1-\sigma_{I}\right) \delta_{C}$ (the incumbent strategy), and the remaining fraction $\epsilon$ adopts strategy $f_{M}=\sigma_{M} \delta_{r^{0}}+\left(1-\sigma_{M}\right) \delta_{C}$ (the mutant strategy), then the average strategy of the population $f_{A}$ is defined as

$$
\begin{aligned}
f_{A} & =\epsilon f_{M}+\bar{\epsilon} f_{I} \\
& =\left(\epsilon \sigma_{M}+\bar{\epsilon} \sigma_{I}\right) \delta_{r_{0}}+\left(\epsilon \bar{\sigma}_{M}+\bar{\epsilon} \bar{\sigma}_{I}\right) \delta_{C} .
\end{aligned}
$$

We shall refer to this game as the simplified Gaussian MAC game. This game has three Nash equilibria: two involving pure strategies $\left(\left(\delta_{r^{0}}, \delta_{C}\right)\right.$ and $\left.\left(\delta_{C}, \delta_{r^{0}}\right)\right)$, and one involving mixed strategies. The latter is $\left(f^{*}, f^{*}\right)$, where $f^{*}=\frac{r^{0}}{C} \delta_{r^{0}}+(1-$ $\left.\frac{r^{0}}{C}\right) \delta_{C}$, which can be verified using Lemma 1 . If a user plays against an opponent who is using strategy $f^{*}$, then he will get the same payoff regardless of his own strategy. Indeed: $\mathcal{U}_{1}\left(\delta_{r^{0}}, f^{*}\right)=\mathcal{U}_{1}\left(\delta_{C}, f^{*}\right)=\mathcal{U}_{1}\left(f, f^{*}\right)=r^{0}$, for all $f \in \Sigma$

This shows that, if the entire population plays $f^{*}$, no single user has an incentive to deviate from this strategy. In the following we prove a stronger property, that no subpopulation of any size has an incentive to deviate from $f^{*}$. We prove this using the concept of evolutionary stable strategy which is central to evolutionary game theory.

Definition 1: $f$ is an evolutionary stable strategy if for every strategy $\hat{f} \neq f$ there exists some $\hat{\epsilon}=\hat{\epsilon}(\hat{f}) \in(0,1]$ such that the inequality

$$
\mathcal{U}_{1}(f, \bar{\epsilon} f+\epsilon \hat{f})>\mathcal{U}_{1}(\hat{f}, \bar{\epsilon} f+\epsilon \hat{f})
$$

holds for all $\epsilon \in(0, \hat{\epsilon})$.

In other words, an evolutionary stable strategy is an incumbent strategy that, when played against an average strategy, earns strictly higher expected payoff than any mutant strategy played by a sufficiently small fraction of the population. Hence, for a population of users playing an evolutionary stable strategy no sufficiently small subgroup will have an incentive to change strategy.

Theorem 3: The mixed strategy $f^{*}=\frac{r^{0}}{C} \delta_{r^{0}}+\left(1-\frac{r^{0}}{C}\right) \delta_{C}$ is the only evolutionary stable strategy for the simple Gaussian MAC game. Furthermore, $\hat{\epsilon}(\hat{f})=1$ for all $\hat{f}$, i.e. users playing $f^{*}$ are earning higher average payoff than the mutants regardless of the size of the mutant population.
Proof: To prove the theorem, we need to show that the strategy $f^{*}$ is strictly better than any other mutant strategy. We begin by checking that $f^{*}$ is better than any strategy of the type $\hat{f}=\left(\frac{r^{0}}{C}-t\right) \delta_{r^{0}}+\left(1-\frac{r^{0}}{C}+t\right) \delta_{C}$, for $t \in\left(0, \frac{r^{0}}{C}\right]$. We suppose that fraction $\epsilon$ of the population plays the mutant strategy $\hat{f}$, and that the remaining $\bar{\epsilon}$ fraction of users stick with the incumbent strategy $f^{*}$. Then,

$$
\begin{gathered}
\mathcal{U}_{1}\left(f^{*}, \bar{\epsilon} f^{*}+\epsilon \hat{f}\right)=r^{0}-\epsilon t C\left(1-\frac{r^{0}}{C}\right) \text { and } \\
\mathcal{U}_{1}\left(\hat{f}, \bar{\epsilon} f^{*}+\epsilon \hat{f}\right)=r^{0}-\epsilon t C\left(1-\frac{r^{0}}{C}+t\right) .
\end{gathered}
$$

So we see that $\mathcal{U}_{1}\left(f^{*}, \bar{\epsilon} f^{*}+\epsilon \hat{f}\right)>\mathcal{U}_{1}\left(\hat{f}, \bar{\epsilon} f^{*}+\epsilon \hat{f}\right)$ for all $t \in$ $\left(0, \frac{r}{C}\right)$, for all $\epsilon \in(0,1)$. This means that, whatever the size of the mutant population, the users playing strategy $f^{*}$ are strictly better off. Similarly, for all $\hat{f}=\left(\frac{r^{0}}{C}+t\right) \delta_{r^{0}}+\left(1-\frac{r^{0}}{C}-t\right) \delta_{C}$, where $t \in\left(0,1-\frac{r^{0}}{C}\right]$ we have:

$$
\begin{gathered}
\mathcal{U}_{1}\left(f^{*}, \bar{\epsilon} f^{*}+\epsilon \hat{f}\right)=r^{0}+\epsilon t C\left(1-\frac{r^{0}}{C}\right) \text { and } \\
\mathcal{U}_{1}\left(\hat{f}, \bar{\epsilon} f^{*}+\epsilon \hat{f}\right)=r^{0}+\epsilon t C\left(1-\frac{r^{0}}{C}-t\right),
\end{gathered}
$$

which shows that $f^{*}$ is an evolutionary stable strategy. Since $f^{*}$ is an interior point (i.e $f^{*} \in \Sigma \backslash\left\{\delta_{r^{0}}, \delta_{C}\right\}$ ) of the set of probability distributions on $r^{0}$ and $C$, we can invoke Proposition 2.2 of [2], which states that an interior strategy that is evolutionary stable is necessarily the unique evolutionary stable strategy of the game.

What Theorem 3 does not concern itself with is how a population gets to playing this strategy. Fortunately, evolutionary game theory has a framework, called replicator dynamics, that investigates the dynamics of the change of the strategies being played by a population.

Replicator dynamics is a system of ordinary differential equations that models how the state of the population is changing, by assuming that the number of offsprings of a user is equal to the payoff of this user in a one-shot game. The assumption is that all the offsprings play the same strategy as their predecessor.

We assume that individuals are programmed to only play pure strategies $r^{0}$ and $C$. We also assume that each of these two strategies is present in the population. Without this assumption, a system that starts in a state where no user plays some pure strategy will necessarily never play that strategy.

Let $N_{r^{0}}>0$ and $N_{C}>0$ denote the number of users playing strategy $r^{0}$ and $C$, respectively, and let $\dot{N}_{r^{0}}$ and $\dot{N}_{C}$ be their time derivatives. The key variable in replicator dynamics is the population state, labeled $\sigma$, where $\sigma=\frac{N_{r 0}}{N_{r 0}+N_{C}}$ is the fraction of users playing strategy $r^{0}$. The fraction of the population playing strategy $C$ is $\bar{\sigma}=1-\sigma$ (In a general setup with $K$ strategies played, the state of the system is an $N$-tuple that indicates the relative frequency of strategies present in the population.). The average strategy of a population with state $\sigma$ is $f=\sigma \delta_{r^{0}}+(1-\sigma) \delta_{C}$. Hence, there is a 1-to-1 map between a population state and its average strategy. 
We assume that the number of offsprings per unit of time of a user is equal to his expected payoff, and that each offspring inherits its (single) parent's pure strategy ${ }^{1}$ :

$$
\begin{aligned}
\dot{N}_{r^{0}} & =\mathcal{U}_{1}\left(\delta_{r^{0}}, f\right) N_{r^{0}}, \\
\dot{N}_{C} & =\mathcal{U}_{1}\left(\delta_{C}, f\right) N_{C},
\end{aligned}
$$

where $f=\sigma \delta_{r^{0}}+(1-\sigma) \delta_{C}$ is the average strategy of a population with state $\sigma$. This model and its analysis are treated in detail in Chapter 3 of [2]. For brevity, we only revisit the key points and equations that are of interest to us. After some manipulations, the fundamental equations of replicator dynamics become:

$$
\begin{aligned}
& \frac{\partial \sigma}{\partial t}=\left(\mathcal{U}_{1}\left(\delta_{r^{0}}, f\right)-\mathcal{U}_{1}(f, f)\right) \sigma \\
& \frac{\partial \bar{\sigma}}{\partial t}=\left(\mathcal{U}_{1}\left(\delta_{C}, f\right)-\mathcal{U}_{1}(f, f)\right) \bar{\sigma} .
\end{aligned}
$$

Therefore, the growth rate of a population share is equal to the difference between the average payoff that the users playing this strategy are getting, and the average population payoff.

It can be proved that (7) and (8) define a continuous solution mapping $\xi: \mathbb{R} \times(0,1) \rightarrow(0,1)$, where $\xi\left(t, \sigma^{0}\right)$ is the state of the population at time $t$, if the initial state at $t=0$ was $\sigma^{0}$.

In general, we are interested in the steady state behavior of the system, i.e. what is the value of $\xi$ as $t \rightarrow \infty$. As it turns out, if the users are playing a game that has an evolutionary stable strategy, the state of the system will converge to one corresponding to this mixed strategy. The statement holds regardless of the initial state. The only condition is that the evolutionary stable strategy is an interior point of the set of mixed strategies (i.e. every pure strategy is played with non-zero probability). This is proved formally in Proposition 3.11 of [2]. The following result is a consequence of this Proposition.

Theorem 4: For any initial state $x^{0}$ of the replicator dynamics described by (7) and (8),

$$
\lim _{t \rightarrow \infty} \xi\left(t, \sigma^{0}\right)=\sigma^{*}
$$

where $\sigma^{*}$ is the state of the system with average strategy $f^{*}=$ $\frac{r^{0}}{C} \delta_{r^{0}}+\left(1-\frac{r^{0}}{C}\right) \delta_{C}$, the unique evolutionary stable strategy of the simple symmetric Gaussian MAC game.

As an example, consider a population whose initial state is $\sigma^{0}<\frac{r^{0}}{C}$. This population has too many users that code at $C$. These users receive a payoff of 0 when playing against each other, and payoff of $C$ when playing against users coding at the safe rate. However, due to the relative frequency of the two strategies, their expected payoff is less than $r^{0}$. On the other hand, the users that code at the safe rate will have a payoff of $r^{0}$, regardless of the system state. In this case, $r^{0}$ is more than the expected average payoff, so $\sigma$ will grow, as predicted by equation (7). By equation (8), the proportion of users that code at capacity will shrink. The rate of growth of $\sigma$

\footnotetext{
${ }^{1}$ The model treated in [2] assumes that a population has an underlying birth rate $\beta$ and death rate $\delta$ which do not depend on the strategies being played. We do not consider these since they do not change anything in our analysis. In particular (7) and (8) hold regardless of the value of $\beta$ and $\delta$.
}

and the rate of decline of $\bar{\sigma}$ will slow down as the state of the system approaches $\sigma^{*}$. The system will eventually converge to the state in which its average strategy is $f^{*}$. On the other hand, a system with the initial state $\sigma^{*}$ will always stay in that state since $\mathcal{U}_{1}\left(\delta_{r^{0}}, f^{*}\right)=\mathcal{U}_{1}\left(\delta_{C}, f^{*}\right)=\mathcal{U}_{1}\left(f^{*}, f^{*}\right)=r^{0}$, so $\frac{\partial \sigma}{\partial t}=0$ and $\frac{\partial \bar{\sigma}}{\partial t}=0$.

\section{CONCLUSION}

In this work we considered the behavior of users in a classical AWGN Multiple Access Channel (MAC). We modeled the interaction of users as a noncooperative one-shot game, in which users were modeled as rational entities whose only interest is to maximize their own communication rate, and the payoffs were the obtained rates. We found the pure strategy, as well as the mixed strategy Nash equilibria of the two-user game. In order to better understand user behavior over time, we have introduced models from evolutionary game theory for a simpler version of the communication game. We found that the population of users will converge to the state in which the average strategy is the strategy that achieves the mixed strategy Nash equilibrium in the simplified one-shot game, defined in Section $\mathrm{V}$, and that the expected payoff is equal to the safe rate.

\section{REFERENCES}

[1] D. Fudenberg and J. Tirole, Game Theory. MIT Press, 1991.

[2] J. Weibull, Evolutionary Game Theory. MIT Press, 1996.

[3] L. Lai and H. El Gamal, "The Water-Filling Game in Fading Multiple Access Channels," ArXiv Computer Science e-prints, 2005.

[4] H. Ji and C.-Y. Huang, "Non-cooperative uplink power control in cellular radio systems," Wirel. Netw., vol. 4, no. 3, pp. 233-240, March 1998.

[5] T. Alpcan, T. Basar, R. Srikant, and E. Altman, "CDMA uplink power control as a noncooperative game," in Proceedings of the 40th IEEE Conference on Decision and Control, vol. 1, 2001, pp. 197-202.

[6] C. U. Saraydar, N. B. Mandayam, and D. J. Goodman, "Efficient power control via princing in wireless data networks," IEEE Transactions on Communications, vol. 50, no. 2, pp. 291-303, February 2002.

[7] F. Meshkati, H. V. Poor, and S. C. Schwartz, "Energy-efficient resource allocation in wireless networks," IEEE Signal Processing Magazine, vol. 24, no. 3, pp. 58-68, May 2007.

[8] N. Bonneau, M. Debbah, E. Altman, and A. Hjorungnes, "Non-atomic games for multi-user systems," ArXiv Computer Science e-prints, June 2007.

[9] N. Bonneau, E. Altman, M. Debbah, and G. Caire, "An evolutionary game perspective to aloha with power control," in Proceedings of the 19th International Teletraffic Congress. Beijing, China, Aug. 29-Sep. 2, 2005.

[10] H. Tembine, E. Altman, and R. El-Azouzi, "Asymmetric delay in evolutionary games," in ValueTools '07: Proceedings of the 2nd international conference on Performance evaluation methodologies and tools, Brussels, Belgium, 2007, pp. 1-8.

[11] R. J. La and V. Anantharam, "A game-theoretic look at the gaussian multiaccess channel," in DIMACS Series in Discrete Mathematics and Theoretical Computer Science, vol. 66, 2004, pp. 87-106.

[12] T. M. Cover and J. A. Thomas, Elements of Information Theory. John Wiley and Sons, 1991. 